\title{
Crack Tip Fields with Strain Gradient Effects
}

\author{
S. H. Chen, T. C. Wang
}

\author{
State Key Laboratory of Nonlinear Mechanics, Institute of Mechanics, Chinese Academy of \\ Sciences, Beijing 100080, China, Email: shchen@lnm.imech.ac.cn
}

\begin{abstract}
In this paper, a kind of strain gradient theory proposed by Chen and Wang (2001) is briefly introduced and is used to analyze the crack tip field in homogeneous materials and interface crack tip field with the help of finite element methods. Differences are found between the two kinds of crack tip fields. For the crack tip field in homogeneous materials, the whole field consists of a strain gradient dominance zone, a classical plasticity field and a $\mathrm{K}$ field. While for the interface crack tip field, there are only the strain gradient dominance zone and a $\mathrm{K}$ field and the classical plasticity field hardly exists. The reason will be given in the present paper. For both kinds of crack tip fields, the effective stresses near the crack tip are much higher than the counterparts in the classical field, which provides an explanation to the experimental observation of cleavage fracture in ductile materials (Elssner et al., 1994).
\end{abstract}

Keywords: Strain gradient effects, fracture, crack tip field

\section{Introduction}

In 1994, Elssner et al. measured both the macroscopic fracture toughness and atomic work of separation of an interface between a single crystal of niobium and a sapphire single crystal. The macroscopic work of fracture was found to be two to three orders of magnitude higher than the atomic work of separation. This large difference between the macroscopic work of fracture and its counterpart at the atomic level was attributed to plastic dissipation in niobium, i.e., there must be significant plastic deformation associated with dislocation activities in niobium. However Elssner et al. (1994) observed that the interface between two materials remained atomistically sharp. Meanwhile the stress level needed to produce atomic decohesion of a lattice or a strong interface is typically on the order of 0.03 times the Young's modulus, or 10 times the tensile yield stress. But the maximum stress level that can be achieved near a crack tip is not larger than 4 or 5 times the tensile yield stress of metals, according to models based on conventional plasticity theories (Hutchinson, 1997). This clearly falls short of triggering the atomic decohesion observed in Elssner et al.'s experiments (1994). Attempts to link macroscopic cracking to atomistic fracture are frustrated by the inability of conventional plasticity theories to model stress-strain behavior adequately at the small scales involved in crack tip deformation.

In order to explain the atomistically sharp crack tip in ductile niobium observed in Elssner et al.'s experiments (1994), it is necessary to develop a continuum theory for micron level. Thus strain gradient theory has been developed. As direct application, strain gradient plasticity theory has been used to investigate fracture of materials. Several significant results (Huang, et al., 1999; Wei and Hutchinson, 1997; Jiang et al. 2001; Chen and Wang, 2002) are obtained. 
In this paper, using the strain gradient theory proposed by Chen and Wang (2001) and finite element method, we investigate crack tip field in homogeneous materials and interface crack tip field in bimaterial. Comparison of the results for the two kinds of crack tip fields are given in this paper.

The strain gradient theory will be briefly given in section 2. Finite element results for crack tip fields in a homogeneous elastic-power law hardening solid are shown in section 3 . Numerical results for the interface crack tip fields will be presented in section 4. Detail discussion is given in section 5 .

\section{Briefly review of the strain gradient theory}

The strain gradient theory (Chen and Wang, 2001) preserves the essential structure of the incremental version of conventional couple stress deformation theory. No higher-order stress or higher-order strain rates are introduced. The rotation gradient influences the material character through the interaction between the Cauchy stresses and the couple stresses; the stretch gradient measures explicitly enter the constitutive relations only through the instantaneous tangent modulus and the boundary value problem of the incremental equilibrium is the same as in the conventional theories. The tangent-hardening modulus is influenced by not only the generalized effective strain but also the effective stretch gradient.

In a Cartesian reference frame $x_{i}$, the strain tensor $\varepsilon_{i j}$ and the stretch gradient tensor $\eta_{y k}$ are related to the displacement $u_{\text {, by }}$

$$
\begin{aligned}
& \varepsilon_{i j}=\frac{1}{2}\left(u_{i, j}+u_{l, l}\right) \\
& \eta_{i j k}=u_{k, j j}
\end{aligned}
$$

The definition $\eta_{i j k}^{(1)}$ can be found in Smyshlyaev and Fleck (1996)

The rotation gradient can be defined as the curvature tensor, which is related with the micro-rotation vectors $\omega_{1}$,

$$
\chi_{i j}=\omega_{i, j}
$$

$$
\varepsilon_{e}=\sqrt{\frac{2}{3} \varepsilon_{i j}^{\prime} \varepsilon_{i j}^{\prime}}, \chi_{e}=\sqrt{\frac{2}{3} \chi_{i j} \chi_{j}}, \eta_{1}=\sqrt{\eta_{i j k}^{(1)} \eta_{j j k}^{(1)}}
$$

In the general couple stress theory, the material rotation vector $\theta_{l}$ is $\theta=(1 / 2)$ curlu . Then the relative rotation tensor $\alpha_{i j}$ is defined as

$$
\alpha_{i j}=e_{y k}\left(\omega_{k}-\theta_{k}\right)
$$

The micro-rotation vector $\omega$, which is the sum of the material rotation vector $\theta$ plus the particle relative rotation vector with respect to the material, is an independent quantity with no direct dependence upon $\boldsymbol{u}$, i.e. $\omega \neq \theta$.

We postulate that the strain energy density $w$ depends only upon the strain tensor $\varepsilon$ and the curvature tensor $\chi$, It follows

$$
\tau_{i j}-\frac{\partial w}{\partial \alpha_{i j}}=0
$$

where $\tau_{\|}$is the anti-symmetric part of Cauchy stress and the work conjugate of the relative rotation tensor $\alpha$.

The deviatoric part $s_{\imath}$ of Cauchy stress and deviatoric part $m_{i j}^{\prime}$ of couple stress are defined as the work conjugates of $\varepsilon_{i j}^{\prime}, \quad \chi_{i j}^{\prime}$ respectively; $\sigma_{m}$ and $m_{m}$ are defined as the work conjugates of $\varepsilon_{m}$ and $\chi_{m}$ respectively, giving

$$
\delta w=s_{i j} \delta \varepsilon_{i j}^{\prime}+m_{i j}^{\prime} \delta \chi_{j}^{\prime}+\sigma_{m} \delta \varepsilon_{m}+m_{m} \delta \chi_{m}
$$

It is mathematically convenient to assume that the strain energy density $w$ depends only upon the single scalar strain measure $E_{e}$, where

$$
E_{e}^{2}==_{e}^{2}+l_{c s}^{2} \ddot{\lambda}_{e}^{2}
$$

where $l_{c s}$ is an intrinsic material length, which reflects the size effects of the rotation gradient on the material behaviors.

$\Sigma_{e}$ is the work conjugate of $E_{e}$, i.e.

$$
\Sigma_{2}=\frac{\mathrm{d} w\left(E_{\mathrm{e}}\right)}{\mathrm{d} E_{z}}
$$

Then the constitutive relations can be written as

Also, we define 
$\sigma_{i j}=s_{i j}+\sigma_{m} \delta_{i j}=\frac{2 \Sigma_{e}}{3 E_{e}} \varepsilon_{i j}^{\prime}+K \varepsilon_{m} \delta_{i j}$

$m_{i j}=m_{i j}^{\prime}+m_{m} \delta_{i j}=\frac{2 \Sigma_{e}}{3 E_{e}} l_{c s}^{2} \chi_{i j}^{\prime}+K_{1} l_{c s}^{2} \chi_{m} \delta_{i j}$

where $\quad \Sigma_{e}=\left(\sigma_{e}^{2}+l_{i}^{-2} m_{e}^{2}\right)^{1 / 2}$

and $K$ is the volumetric modulus and $K_{\text {: }}$ is called the bend-torsion volumetric modulus.

While the stretch gradient is not considered, the hardening relation between $\Sigma_{e}$ and $E_{e}$ in this paper is taken as

$$
\begin{cases}\Sigma_{e}=A\left(E_{e}\right), & \Sigma_{\mathrm{e}}>\sigma_{Y} \\ \Sigma_{e}=3 \mu E_{e}, & \Sigma_{\mathrm{e}}<\sigma_{Y}\end{cases}
$$

While the stretch gradient is considered, the incremental hardening relationship is proposed

$$
\begin{cases}\dot{\Sigma}_{e}=A^{\prime}\left(E_{e}\right)\left(1+\frac{l_{1} \eta_{1}}{E_{e}}\right)^{\frac{1}{2}} \dot{E}_{e}, & \Sigma_{e}>\sigma_{Y} \\ \dot{\Sigma}_{e}=3 \mu \dot{E}_{e} & \Sigma_{e}<\sigma_{Y}\end{cases}
$$

where $\mu$ is the shear modulus and $l_{1}$ is the second intrinsic material length.

In order to solve the crack tip field, the constitutive relations are written in the incremental form also.

$$
\begin{aligned}
& \left\{\begin{array}{l}
\dot{\sigma}_{i j}=2 \mu \dot{\varepsilon}_{i j}^{\prime}+K \dot{\varepsilon}_{m} \delta_{i j} \\
\dot{m}_{i j}=2 \mu l_{c s}^{2} \dot{\chi}_{i j}^{\prime}+K_{1} l_{c s}^{2} \dot{\chi}_{m} \delta_{i j}, \quad \Sigma_{e}<\Sigma_{0}
\end{array}\right. \\
& \left\{\begin{array}{l}
\dot{\sigma}_{i j}=\frac{2 \Sigma_{e}}{3 E_{e}} \dot{\varepsilon}_{i j}^{\prime}+\frac{2 \dot{\Sigma}_{e}}{3 E_{e}} \dot{c}_{i j}^{\prime}-\frac{2 \Sigma_{s}}{3 E_{g}^{2}} \varepsilon_{i j}^{\prime} \dot{E}_{e}+K \dot{\varepsilon}_{m} \delta_{i j} \\
\dot{m}_{i j}=\frac{2 \Sigma_{e}}{3 E_{e}} l_{c s}^{2} \dot{\chi}_{i j}^{\prime}+\frac{2 \dot{\Sigma}_{e}}{3 E_{e}} l_{c s}^{2} \dot{\chi}_{i j}^{\prime}- \\
\frac{2 \Sigma_{e}}{3 E_{e}^{2}} l_{c s}^{2} \chi_{i j}^{\prime} \dot{E}_{e}+K_{1} l_{c s}^{2} \dot{\chi}_{m} \delta_{i j}
\end{array},\right.
\end{aligned}
$$

\section{Crack tip field in homogeneous materials}

9-node element is used in this paper. The displacement and rotation vectors in the element are interpolated through the shape function. Plane strain case is studied in the present paper. The domain for the finite element analysis is a circle, whose central point is at the crack tip and the radius is $R=1000 l_{c s}$. The classical $\mathrm{K}$ fields are imposed on the outer boundary. The results presented below were computed with $\sigma_{Y} / E=0.2 \%, \quad v=0.3$, $R=1000 l_{\text {.... }}$.

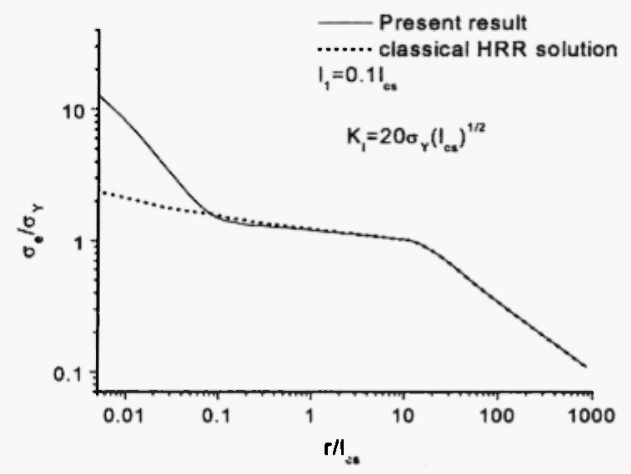

Fig.1. Normalized effective stress ahead of the crack tip versus the normalized distance

Fig.l shows the normalized effective stresses, $\sigma_{e} / \sigma_{Y}$, at polar angle $\theta=0^{0}$ versus the normalized distance $r / l_{c s}$ for the present strain gradient theory with the plastic hardening exponent $n-0.2$ and $l_{1}=0.1 l_{c s}$. The remotely applied stress intensity factor in Fig.l is $K_{l} /\left(\sigma_{Y} l_{c s}^{1 / 2}\right)=20$. The plastic zone size is a bit more than $10 l_{c s}$. The corresponding stress distribution in classical plasticity (without strain gradient effects) is also shown in Fig. 1. Outside the plastic zone, it is observed that both the present strain gradient theory and the classical plasticity theory give the same straight line with slope $-1 / 2$, which corresponds to the elastic $K$ field. The predictions of the present strain gradient theory and the classical plasticity theory are almost the same within the plastic zone at a distance larger than $0.06 l_{c s}$ to the crack tip. At a distance of $0.03 l_{c s}$ to the crack tip, the effective stress given by the present strain gradient theory is much higher than that in classical plasticity and the absolute value of 
the slope is larger than that for the HRR field, which means that the stresses around the crack tip in the present strain gradient theory are more singular than the HRR field. The order of the stress singularity nearly tends to be $-1 / 2$.

In Fig.2, materials with various hardening exponents are calculated and the effects of the hardening exponents on the effective stress distribution ahead of the crack tip are shown in Fig.2. The remotely applied stress intensity factor is $K_{I} /\left(\sigma_{Y} l_{c s}^{1 / 2}\right)=10$ also and $l_{1}=0.1 l_{c s}$. The hardening exponents are $n=0.1,0.2,0.33$. One can find from Fig. 2 that near the crack tip there is a domain dominated by the strain gradient, the slope is hardly related to the hardening exponents and nearly the same as that of classical $\mathrm{K}$ field.

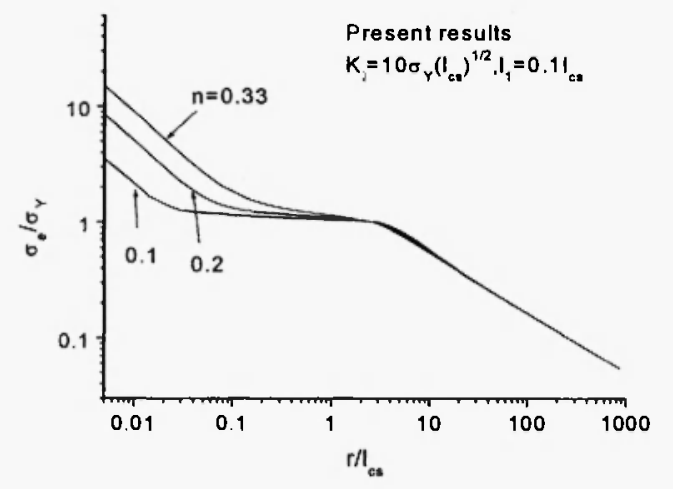

Fig.2. The results for different hardening exponents with the same external loading

In this paper, the main object is to compare the results for the crack tip field in homogeneous materials and those for an interface crack. So some other phenomena are not shown and can be found in Chen and Wang (2002).

\section{Interface crack tip field}

The interface crack with a rigid substrate is often found in engineering problems and here this special kind of case is calculated and the strain gradient is considered. A finite square plate subject to uniform tensile is considered and the calculation model is shown in Fig.3, in which only the right half of the deformable medium is considered. The half of the crack length is $a$ and the width of the calculated model is $10 a$. On the boundary of $y=10 a$, only normal stress $\sigma_{22}^{\infty}$ is imposed. During the calculation the parameters of the upper material are $\sigma_{y} / E=0.2 \%, v=0.3, n=0.2$. In all the calculation, we take $l_{c s}=1 \mu \mathrm{m}$.

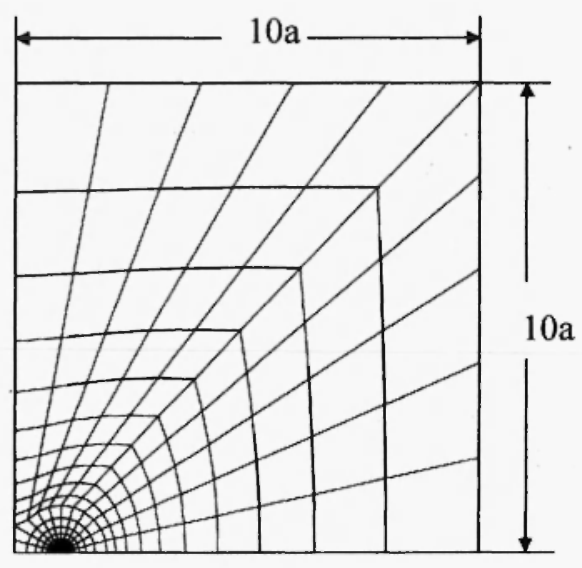

Fig.3 Mesh division for interface crack

Fig.4 shows the normalized effective stresses, $\sigma_{e} / \sigma_{Y}$, versus the non-dimensional distance to the crack tip, $r / l_{c s}$, ahead of the crack tip $\left(\theta=3.15^{\circ}\right)$. The remotely applied stress is $\sigma_{: 2}^{\infty} / \sigma_{Y}=1 / 6$. There are four kinds of cases in Fig.4, in which three results are corresponding to different relations of $l_{c s}$ and $l_{1}$, the other one is for the classical theory. Since the material length $l_{c s}$ is taken prior, the value of $l_{1}$ for each case is known also. The horizontal line separates the elastic and plastic zones for each curve. Outside the plastic zone and $r / l_{c s}<5$, both the present strain gradient theory and the classical plasticity theory give the same straight line with slope $-1 / 2$, which corresponds to the elastic K field. As $r / l_{c s}>5$, there is an elastic field, which is influenced by the outer boundary. From Fig.4, one can find the interface crack tip field is significantly influenced the material length $l_{1}$ while $l_{1}>0.01 \mu \mathrm{m}$. When $l_{1}>0.1 \mu \mathrm{m}$, the HRR type field seems to vanish, there is no HRR type field, which is different from the results for crack tip field in homogeneous material. This interesting phenomena may be due to the 
intrinsic properties of the interface crack between and elastic-plastic solid and a rigid substrate. Since the lower material is rigid substrate, the material points along interface in both directions are fixed, which results in the greater strain gradient along $y$ direction near the interface. While the value of material length $l_{1}$ decrease, the result tends to the classical solution, which reflects that the length scale $l_{1}$ plays an important role in crack tip field.

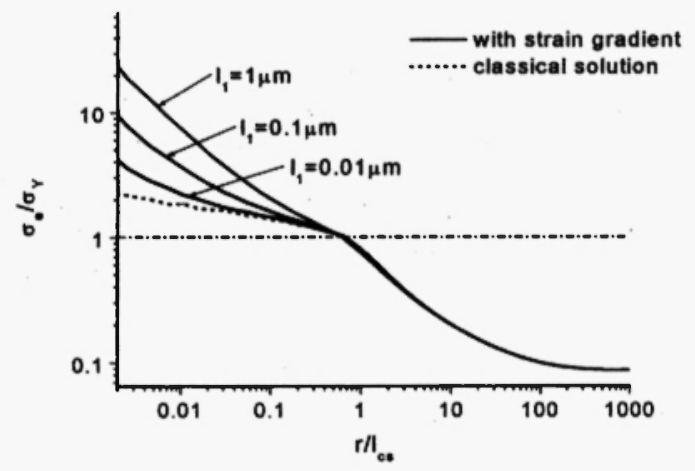

Fig.4 Normalized effective stresses ahead of an interface crack for different $l_{1}$

\section{Discussion}

For the crack tip field in homogeneous materials, the full field solutions are obtained numerically with remotely imposed classical $\mathrm{K}$ fields. It is found that the size of the strain gradient dominance zone is characterized by the intrinsic material length $l_{1}$. Outside the strain gradient dominance zone, the computed stress field tends to be a classical plasticity field and then $\mathrm{K}$ field. The singularity of stresses ahead of the crack tip is higher than that of the classical field and tends to the square root singularity. The effective stress near the crack tip is significantly improved and much higher than the counterpart in the classical theory.

As for the interface crack tip field, under small scale yielding condition and $l_{1}>0.1 \mu \mathrm{m}$, the remote classical $\mathrm{K}$ field goes directly to the near tip strain gradient dominated zone without a classical plasticity field. The material length scale $l_{1}$ has an important influence on the interface crack tip field and once the plasticity is produced, the effects of strain gradient dominate the field, which is different from that in homogeneous material.

For both kinds of crack tip filed, at a distance that is much larger than the dislocation spacing such that continuum plasticity is expected to be applicable. The near tip stresses predicted by the strain gradient theory are significantly higher than that in HRR field. The increase in the near tip stress level provides an explanation to the experimental observation of cleavage fracture in ductile materials.

\section{Acknowledgements}

This work is supported by the NSF of CAS and Excellent Post-doctoral Research-Starting Fund of CAS.

\section{References}

[1] Chen, S.H., Wang, T.C., A new hardening law for strain gradient plasticity, Acta Materialia 48, (2000)3997-4005

[2] Chen, S.H., Wang, T.C., Strain gradient theory with couple stress for crystalline solids, European J. Mech. A/Solids, 20(2001)739-756

[3] Chen, S.H., Wang, T.C., Finite element solutions for plane strain mode I crack with strain gradient effects, Int. J. Solids Struct, 39(5)(2002)1241-1257

[4] Elssner, G., Korn, D., Ruehle, M., The influence of interface impurities on fracture energy of UHV diffusion bonded metal-ceramic bicrystals, Scripta Metall. Mater. 31(1994)1037-1042

[5] Huang, Y., Chen, J.Y., Guo, T.F., Zhang, L., Hwang, K.C., Analysis and numerical studies on mode I and mode II fracture in elastic-plastic materials with strain gradient effects, Int. J. Fracture 100(1999)1-27

[6] Hutchinson, J.W., Linking scales in mechanics. In: Karihaloo, B. L., Mai, Y. W., Ripley, M. I., Ritchie, R. O. (Eds), Advances in fracture research. Pergamon Press, Amsterdam, pp.l14(1997)

[7] Jiang, H., Huang, Y., Zhuang, Z., Hwang, K.C., Fracture in mechanism-based strain gradient plasticity, J. Mech. Phys. Solids 49(2001)979993

[8] Wei, Y., Hutchinson, J.W., Steady-state crack growth and work of fracture for solids characterized by strain gradient plasticity, J. Mech. Phys. Solids 45(1997)1253-1273 